\title{
Acute joint inflammation alters the adrenoceptor profile of synovial blood vessels in the knee joints of rabbits
}

\author{
E Gray, W R Ferrell
}

\begin{abstract}
Experiments were carried out to examine the effect of acute inflammation, induced by intra-articular injection of $2 \%$ carrageenan, on the response of articular blood vessels in the knee joints of rabbits to adrenoceptor agonists. The responses to noradrenaline, phenylephrine, clonidine, UK-14304, and isoprenaline were examined 24 hours after carrageenan injection and compared with those of normal animals. Antagonists specific for $\alpha_{1}$ and $\alpha_{2}$ were used to identify the adrenoceptors through which the responses were mediated and to examine if carrageenan treatment altered the adrenoceptor profile of these blood vessels. The evidence suggests that in the carrageenan treated animals there is a reduction in the $\alpha_{1}$ response with an associated increase in the $\alpha_{2}$ response. A decrease in the number or affinity of $\alpha_{1}$ adrenoceptors is indicated by the shift to the right of the noradrenaline and phenylephrine dose/response curves, whereas an increase in $\alpha_{2}$ affinity or number is suggested by the associated leftward shift in the $\alpha_{2}$ adrenoceptor agonist curves. This change in receptor profile appears to arise as a direct result of carrageenan induced joint inflammation.
\end{abstract}

(Ann Rheum Dis 1992; 51: 1129-1133)

The regulation of synovial blood flow is of critical importance to the maintenance of a stable intra-articular environment. Vessel calibre in numerous vascular beds has been shown to be under humoral and neural control. Although there have been few studies investigating the control of blood flow within the synovium, evidence is beginning to emerge indicating that these two factors play a part in controlling synovial blood flow. Synovial vessels in the knee joints of rabbits respond to hormonal and neural stimuli, resulting in vasoconstriction which is mediated via $\alpha$ adrenoceptors present on and within the vascular smooth muscle. ${ }^{1-3}$ Additionally, human and animal synovium have been shown to be richly innervated by autonomic nerve fibres and sensory nerves containing neuropeptides such as substance $P$ and calcitonin gene related peptide,${ }^{4}$ which are potent dilators vium has been shown in tissue obtained from patients with rheumatoid arthritis. ${ }^{4}$ In addition, a reduction in the neuropeptide content of the nerve fibres in deeper tissues was also observed. These factors could affect the hyperaemia associated with joint inflammation.

Thus the presence of adrenoceptors and innervation of synovial tissue suggests that hormonal and neural factors affect the regulation of blood flow. Vasodilator and vasoconstrictor agents, either neurally released or circulating in the blood, act together to maintain a homeostatic balance which is reflected in the calibre of the vessels. In inflamed tissues, changes in nerve fibre number, the amount of neurotransmitter released, or of binding ability (affinity/receptor number) could alter this control mechanism. This study attempts to examine the adrenoceptor profile of blood vessels in the knees of rabbits during acute inflammation to assess whether changes in receptor profile or affinity occur and if this is related to the vasodilatation known to occur in joint inflammation.

\section{Materials and methods}

Acute inflammation was induced in the knee joints of albino New Zealand rabbits (either sex, $2-4 \mathrm{~kg}$ ) using a $2 \%$ solution of carrageenan (Sigma). The rabbits were anaesthetised intramuscularly with Hypnorm (Janssen) $(1 \mathrm{mg}$ ) and a $0.1 \mathrm{ml}$ bolus of carrageenan was injected into each knee, ensuring that the needle had penetrated the knee space before injecting a portion of the solution. The needle was then withdrawn slightly before injecting the remaining solution, ensuring that carrageenan was distributed throughout the synovial space. Visual inspection of the knee during the experiment allowed this to be confirmed. The animal was then allowed to recover. Approximately 24 hours after injection the animal was killed with a blow to the skull followed by exsanguination. Immediately afterwards the posterior aspect of the knee was exposed, the muscular branches of the popliteal artery ligated, and the artery cannulated as described previously. ${ }^{1}$ Before placing the preparation in the tissue chamber the capsule was flushed with saline to identify any major leakages, which were then ligated.

The tissue was then transferred to a chamber filled with Locke's solution $(\mathrm{NaCl} 115 ; \mathrm{KCl} 4 \cdot 7$; $\mathrm{CaCl}_{2} 2 \cdot 5 ; \mathrm{MgSO}_{4} \cdot 7 \mathrm{H}_{2} \mathrm{O} 24 \cdot 1 ; \mathrm{KH}_{2} \mathrm{PO}_{4} 1 \cdot 2$, and glucose $5.6 \mathrm{mmol} / \mathrm{l})$. Calcium chloride was added after oxygenating the solution with $95 \%$ $\mathrm{O}_{2}$ and $5 \% \mathrm{CO}_{2}$. The chamber was thermostatically maintained at $35 \pm 1^{\circ} \mathrm{C}$ and the preparation perfused at a known rate with \footnotetext{
of articular blood vessels. ${ }^{5}$

Inflammatory diseases such as rheumatoid arthritis result in increased blood flow in the affected joint but the mechanisms responsible are poorly understood. There is evidence to suggest that there is a neurogenic component in animal models of joint inflammation. ${ }^{6}$ Depletion of the nerves innervating the superficial syno-

Correspondence to: Dr Ferrell.

The University,

Glasgow G12 8QQ,

United Kingdom

E Gray
} 
oxygenated Locke's solution using a peristaltic pump (Gilson Miniplus, $3.0 \mathrm{rev} / \mathrm{min}$ ). An indirect measurement of vessel calibre was established using a pressure transducer attached downstream from the pump (fig 1). As the pump perfusion rate was kept constant, the pressure changes occurring with drug administration reflected the variation in the degree of constriction of the blood vessels. Subsequent changes in pressure as a result of treatment were compared with the baseline perfusion pressure (control) and expressed as the maximum percentage change. After 30 minutes, to allow the preparation to stabilise, the capsule was treated with the vasoactive agent under investigation.

The drugs were added as $0.1 \mathrm{ml}$ bolus injections via tubing connected to the cannula; all drugs were made up in Locke's solution with $0.5 \mathrm{ml}$ ethanol added to the anhydrous powder where appropriate (noradrenaline hydrochloride, clonidine, isoprenaline sulphate, and phenylephrine hydrochloride (Sigma); UK-14304 ditartrate[5-bromo-6(2-imidazolin2-ylamino)-quinoxaline] (Pfizer)). Periodically, 'control' injections of Locke's solution were administered to ensure that the responses seen were not artefacts caused by the method of administration. When used, the antagonists were continually perfused with prazosin hydrochloride (Pfizer) and rauwolscine hydrochloride (Roth) made up in Locke's solution.

The data are presented as mean (SEM). Comparison of means was by paired or unpaired $t$ test as appropriate.

\section{Results}

NORADRENALINE

Intra-arterial injection of noradrenaline resulted in a dose dependent vasoconstriction of knee joint blood vessels in the normal animals and in those treated with carrageenan (fig 2A). The dose response profiles follow a similar pattern, but in the carrageenan group there is a shift to the right with significant deviations at higher doses $\left(1 \times 10^{-9}-1 \times 10^{-7} \mathrm{~mol}\right)$. In addition, this figure incorporates the effect of prazosin perfusion $\left(1 \times 10^{-6} \mathrm{~mol} / \mathrm{l}\right)$ in the normal and carrageenan groups (fig 2B). In the normal animals responses were reduced at all doses when compared with controls (fig 2A), whereas in the animals treated with carrageenan the responses were completely abolished at the lower doses and reduced at higher doses. Although administration of the $\alpha_{2}$ antagonist rauwolscine resulted in a slight reduction of the response to noradrenaline in normal animals (fig 2C) compared with controls (fig 2A), this difference was not significant $(p=0 \cdot 11 ; n=5)$ and was much less potent than the $\alpha_{1}$ antagonist prazosin (fig 2B), which produced a significant reduction $(p=0.022 ; n=5)$. Comparison of the responses during prazosin and rauwolscine

Figure 1 Schematic diagram of the preparation of the isolated, perfused rabbit knee joint.

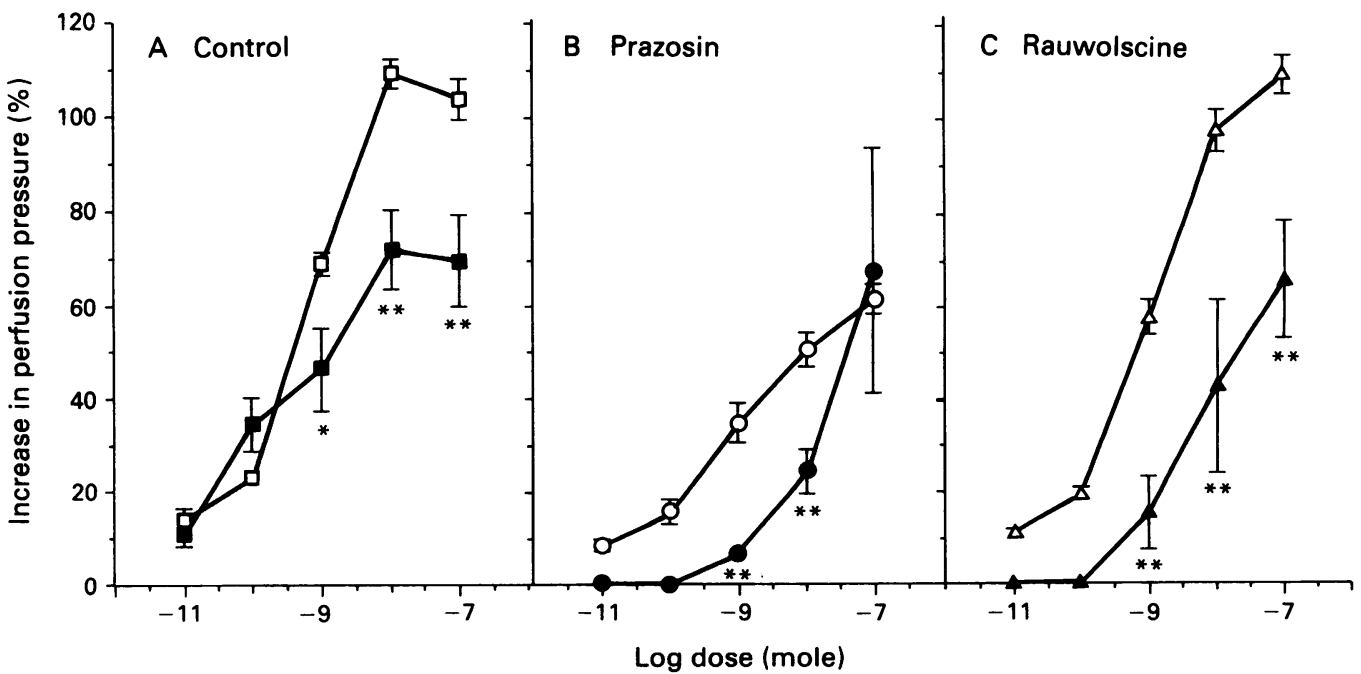

Figure 2 Change in perfusion pressure (mean $(S E M))$ with increasing dose of noradrenaline in normal $(\square, \bigcirc, \triangle ; n=6-12)$ and carrageenan treated $(\square, 0, \Delta ; n=8-11)$ animals $(A)$ under control conditions, $(B)$ during prazosin perfusion $\left(1 \times 10^{6}\right.$ molll, and $(C)$ during rauwolscine perfusion $\left(1 \times 10^{5}\right.$ moll/l). Statistical significance refers to normal $v$ carrageenan treated animals in each graph. ${ }^{*} p \leqslant 0 \cdot 05,{ }^{* *} p \leqslant 0 \cdot 01$. 
perfusion showed significant differences $(p=0.032 ; n=5)$. In the carrageenan treated group prazosin and rauwolscine were effective in significantly reducing the constrictor response to noradrenaline $(p=0.018$ and $\mathrm{p}=0.022$ respectively compared with the control; $\mathrm{n}=5$ ), especially at the lower doses. Comparison of the prazosin and rauwolscine treated groups showed no significant difference $(p=0 \cdot 12$; $n=5$ ). In all three instances the profile of the carrageenan treated animals appears to have moved to the right, suggesting that there is a shift in the $\alpha_{1}$ constituent of the curve. Therefore it appears that the response to noradrenaline is mediated principally through $\alpha_{1}$ adrenoceptors in normal animals with carrageenan treatment altering the $\alpha_{1}$ component of the curve, thereby moving the response curve to the right, and concomitant enhancement of the $\alpha_{2}$ component.

PHENYLEPHRINE, CLONIDINE, AND UK-14304 To investigate further if carrageenan treatment alters $\alpha_{1}$ adrenoceptors, the effect of the selec-

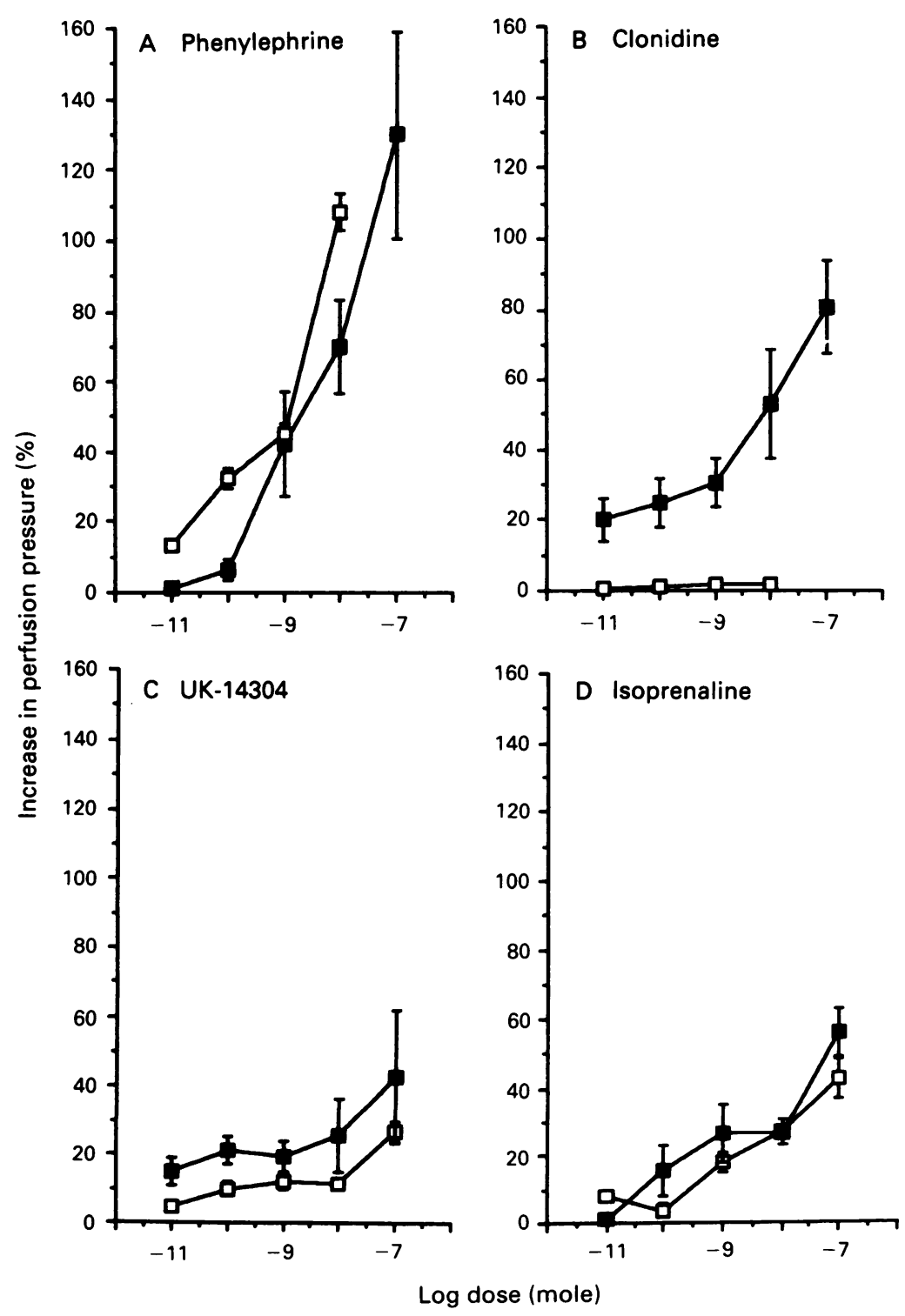

Figure 3 Change in perfusion pressure in normal $(\square)$ and carrageenan treated animals ( in response to $(A)$ phenylephrine $(n=6-12),(B)$ clonidine $(n=5-11),(C) U K-14304$, $(n=5-10)$, and $(D)$ isoprenaline $(n=6-10)$. Data presented as mean $(S E M)$. tive $\alpha_{1}$ agonist phenylephrine was compared with the $\alpha_{2}$ agonists clonidine and UK-14304. Administration of phenylephrine resulted in a similar trend in normal and carrageenan treated animals: an increase in vasoconstriction with increasing dose (fig 3A). Once again, however, the dose response curve is shifted slightly to the right in the carrageenan group.

In the normal group clonidine produced almost no response, whereas in the carrageenan group a distinct dose dependent vasoconstriction occurred (fig 3B). This difference was statistically significant $(p \leqslant 0 \cdot 01)$ at all doses $(n=6-12)$. UK-14304 treatment resulted in a constrictor response in the normal and carrageenan groups (fig 3C). The treated animals showed the same trend but of a slightly higher magnitude at all doses; however, this was not of the same degree as clonidine. In the two instances the response curves shifted to the left, suggesting that there is an alteration in the $\alpha_{2}$ receptor profile, either an increase in number or affinity resulting from carrageenan treatment.

ISOPRENALINE

Previously isoprenaline has been shown to have a constrictor effect mediated by cross reactivity with $\alpha$ adrenoceptors. ${ }^{1}$ In this study the two groups show similar vasoconstrictor responses with no significant difference occurring (fig 3D).

\section{PRAZOSIN}

The effect of prazosin perfusion at $1 \times 10^{-6} \mathrm{~mol} / 1$ was examined in the animals treated with carrageenan (perfusion for a minimum of one hour). This concentration was selected because it has previously been shown to reduce $\alpha_{1}$ mediated responses in the normal animal. ${ }^{1}$ Figure 4A shows the effect of prazosin perfusion $\left(1 \times 10^{-6} \mathrm{~mol} / \mathrm{l}\right)$ on noradrenaline, phenylephrine, UK-14304, and clonidine, all injected at a dose of $1 \times 10^{-8} \mathrm{~mol}$. The noradrenaline and phenylephrine responses were significantly reduced to approximately 62 and $55 \%$ respectively, indicating the involvement of $\alpha_{1}$ adrenoceptors in their constrictor effects $(p \leqslant 0.05 ; n=7-10)$. Similar effects occurred at $1 \times 10^{-9} \mathrm{~mol} / \mathrm{l}$ but with a greater percentage reduction, whereas at $1 \times 10^{-10} \mathrm{~mol} / \mathrm{l}$, blockage was complete. The responses to clonidine and UK-14304 were not significantly reduced by prazosin infusion.

\section{RAUWOLSCINE}

Rauwolscine perfusion $\left(1 \times 10^{-5} \mathrm{~mol} / \mathrm{l}\right)$ was also performed for a minimum of one hour before injection of the agonists (fig 4B). The constrictor response to noradrenaline injection was significantly reduced during rauwolscine perfusion, indicating that $\alpha_{2}$ adrenoceptors partially mediate this response. The response to phenylephrine, however, was not significantly reduced by rauwolscine treatment. The responses to UK-14304 and clonidine were significantly reduced by rauwolscine perfusion $(p \leqslant 0.05$ $n=5-7 ; p \leqslant 0.01 ; n=7$ respectively) indicating the important part $\alpha_{2}$ receptors play in mediating 


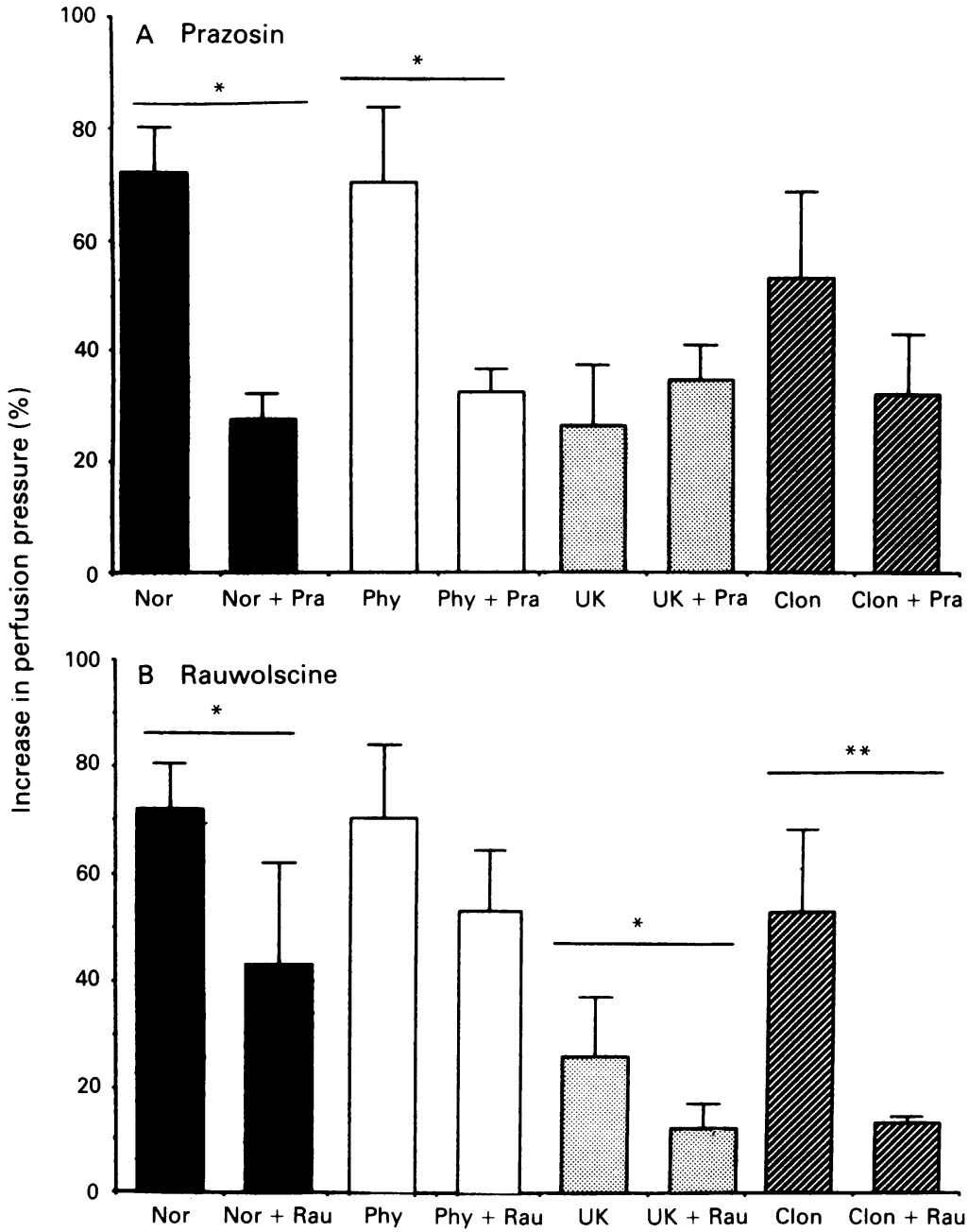

Figure 4 Comparison of the effect of prazosin $\left(1 \times 10^{-6} \mathrm{~mol} / \mathrm{l}\right)$ and rauwolscine $\left(1 \times 10^{5}\right.$ $\mathrm{mol} / \mathrm{l})$ perfusion in the carrageenan treated group on the response to close intra-arterial injection of noradrenaline (Nor; $\left.1 \times 10^{8} \mathrm{~mol}, n=6-11\right)$, phenylephrine $\left(\mathrm{Phy} ; 1 \times 10^{-8}\right.$ mol, $n=4-12), U K-14304$ ( $U K ; 1 \times 10^{-8}$ mol, $\left.n=4-10\right)$, and clonidine (Clon; $1 \times 10^{8}$ mol, $n=4-11)$. Data presented mean $(S E M)$. " $p \leqslant 0 \cdot 05 ; ": p \leqslant 0 \cdot 01$

$\alpha$ adrenoceptor constrictor responses in carrageenan treated animals.

\section{Discussion}

The results presented here support the view that within synovial blood vessels noradrenaline and phenylephrine act via $\alpha_{1}$ adrenoceptors, ${ }^{1}$ and in normal and carrageenan treated animals these agents cause vasoconstrictor responses which are sensitive to prazosin. In addition, the significant reduction in the noradrenaline response following rauwolscine perfusion in the carrageenan treated animals indicates that $\alpha_{2}$ adrenoceptors play a part in mediating this response, unlike the phenylephrine response which is mediated entirely by $\alpha_{1}$ adrenoceptors. In both instances, however, the carrageenan dose response curve is shifted to the right, indicating that there has been some change in the adrenoceptor profile of the tissue. Therefore, it appears that acute inflammation alters the affinity or number of $\alpha$ adrenoceptors. A comparison of the effect of prazosin perfusion in normal and carrageenan treated animals shows that at all but the highest dose of noradrenaline there is a significant reduction from that of the control animals, suggesting that the $\alpha_{1}$ com- ponent has been altered. From this evidence it appears that in normal animals $\alpha_{1}$ receptors form a large proportion of the adrenoceptor profile, whereas in the carrageenan treated animals the $\alpha_{1}$ and $\alpha_{2}$ subtypes are equally abundant. Earlier work by Khoshbaten and Ferrell suggested that postjunctional $\alpha$ adrenoceptors can be altered in some manner, the receptors perhaps becoming more sensitive after kaolin induced joint inflammation. ${ }^{7}$ If a contractile response is associated with a receptor reserve then this reserve buffers the tissue from the effect of inhibitory stimuli; however, in the absence of, or assuming a reduction in, this reserve (that is, the number of receptors), the response will be more sensitive to inhibition, ${ }^{8}$ as shown here. Therefore this evidence, combined with the shift in the curve, points towards a reduction in the $\alpha_{1}$ constituent of the response in the inflamed joint.

Prolonged exposure to a wide range of drugs or hormones may result in an attenuation of physiological responses. Decreased responsiveness may be accompanied by a decrease in receptor number. Decreased responsiveness without an associated decrease in receptor number may also occur, however, perhaps by some form of desensitisation or reduction in receptor number accounting for an altered response. ${ }^{910}$ If this is so then the opposite may also be true and thus the significantly increased constrictor effect of clonidine may be due to sensitisation, upregulation, or an increase in receptor number. The response to clonidine in the carrageenan treated animals is not affected by prazosin but is reduced at higher doses by rauwolscine, indicating that the $\alpha_{2}$ adrenoceptors are mediating the constrictor response. There is, however, no significant increase in the UK14304 response, although this too is mediated via $\alpha_{2}$ receptors as rauwolscine blockade also has an effect. Evidence from ligand binding suggests that $\alpha_{2}$ adrenoceptors show heterogeneity. "12 Gene cloning techniques suggest that there are at least two genes coding for $\alpha_{2}$ adrenoceptors. ${ }^{13}$ Therefore it may be that clonidine and UK-14304 act via $\alpha_{2}$ receptors but the mechanism of action is mediated via different subtypes and that only the $\alpha_{2}$ subtype mediating the clonidine response is significantly affected by carrageenan treatment. In addition, this suggests that noradrenaline and UK-14304 act through the same receptor subtype, as the $\alpha_{2}$ component of the noradrenaline response does not appear to be increased. This hypothesis cannot be substantiated with the current evidence, however.

Alternatively, it has been shown that in relatively high doses clonidine stimulates $\alpha_{1}$ receptors causing directly mediated vasoconstriction. ${ }^{14}$ It is therefore possible that in addition to reducing the receptor number carrageenan makes the remaining $\alpha_{1}$ receptors more sensitive to clonidine. The lack of effect of prazosin on the clonidine response, however, suggests that this 'sensitivity' is in no way significant. This alteration in $\alpha_{1}$ sensitivity to clonidine would not affect the response to UK14034 as this agent is recognised to be more specific for $\alpha_{2}$ adrenoceptors. 
The significant increase in response to clonidine and the associated increased magnitude of the UK-14034 response, although not significant, supports the view that there has been some alteration in the adrenoceptor profile. This change possibly represents a reduction in the number of $\alpha_{1}$ receptors with an increase in the $\alpha_{2}$ number or affinity. The shift to the right for the noradrenaline and phenylephrine response curves and the associated sensitivity to prazosin indicates a reduction in the number of $\alpha_{1}$ receptors, thereby reducing the response at the higher doses.

In conclusion, the results suggest that in the carrageenan treated animals there is a change in the receptor profile, either an alteration in the affinity or a change in the proportion of the receptors. Within 24 hours of inducing acute inflammation, a reduction in the $\alpha_{1}$ adrenoceptor number occurs with an associated increase in $\alpha_{2}$, either with respect to number or affinity. In addition, the evidence points to either a sensitisation of the remaining $\alpha_{1}$ receptors to clonidine or that clonidine and UK-14304 function through different $\alpha_{2}$ subtypes, carrageenan significantly increasing the response to clonidine but not to UK-14304. If this is so, then noradrenaline and UK-14304 function through the same $\alpha_{2}$ subtype. Therefore these results infer that in inflammatory diseases a subtle change in adrenoceptor profile occurs.

These findings may be of clinical relevance as it has been shown that knee joint blood vessels in patients with rheumatoid arthritis appear to lack sympathetic vasoconstrictor 'tone'. 15 The results of this study indicate that over a relatively short period there is a shift in the adrenoceptor profile from the $\alpha_{1}$ to the $\alpha_{2}$ subtype. It is possible that this represents only the early stages of a process of altered adrenoceptor expression and that with chronic joint inflammation this process could proceed further still, perhaps resulting in the expression of receptors with a lower affinity for their naturally occur- ring ligand, noradrenaline. Reduction of sympathetic vasoconstrictor tone in the inflamed joint could therefore contribute to the hyperaemia associated with inflammation and thereby enhance this process.

This research was supported by the Arthritis and Rheumatism Council.

1 Ferrell W R, Khoshbaten A. Adrenoceptor profile of blood vessels in the knee joint of the rabbit. $\mathcal{f}$ Physiol (Lond) 1989; 414: 377-83.

2 Ferrell W R, Khoshbaten A. Responses of blood vessels in the rabbit knee joint to electrical stimulation of the joint the rabbit knee joint to electrical stimulation

3 Cobbold A F, Lewis D J. The nervous control of joint blood vessels. F Physiol (Lond) 1956; 133: 467-71.

4 Mapp P I, Kid D B L, Gibson S J, et al. Substance P. calcitonin gene related peptide- and C-flanking peptide of neuropeptide $\mathrm{Y}$-immunoreactive fibres are present in normal synovium but depleted in patients with rheumatoid arthritis. Neuroscience 1990; 37: 143-53.

5 Lam F Y, Ferrell W R. CGRP modulates nerve-mediated vasoconstriction of rat knee joint blood vessels. Ann $N Y$ Acad Sci 1992; 657: 519-21.

6 Lam F Y, Ferrell W R. The neurogenic component of different models of acute inflammation in the rat knee joint. Ann Rheum Dis 1991; 50: 747-51.

7 Khoshbaten A, Ferrell W R. Responses of blood vessels in the rabbit knee to acute joint inflammation. Ann Rheum Dis $1990 ; 49: 540-4$

8 Flavahan N A, Vanhoutte P M. Receptor reserve and heterogeneity of vascular responses to vasodilator stimuli. Vasodilatation. New York: Raven Press, 1988: 201-10.

9 Ishii K, Kato R. Development of tolerance to alpha adrenergic agonists in the vascular system of the rat after chronic treatment with clonidine. $\mathcal{J}$ Pharmacol Exp Ther 1984; 23 685-91.

10 Brodde O-E, Anlanf M, Graben N, Bock K D. In vitro and in vivo down-regulation of human platelet alpha ${ }_{2}$ adrenoceptors by clonidine. Eur 7 Clin Pharmacol 1982; 23: 403-9.

11 Alabaster V A, R F, Keir C J. Peters comparison of the potency of $a_{2}$ adrenoceptor antagonists in vitro; evidence potency of $\alpha_{2}$ adrenoceptor antagonists in vitro; evidence 1986; 88: 607-14.

12 Connaughton S, Docherty J R. Functional evidence for heterogeneity of peripheral prejunctional $\alpha_{2}$-adrenoceptors Br F Pharmacol 1990; 101: 285-90.

13 Regan J W, Kobilka T S, Yang-Feng T L, Caron M G Lef-Kowitz R J, Kobilka B K. Cloning and expression of a human kidney cDNA for an $(\ell$-adrenergic receptor subtype. Proc Natl Acad Sci USA 1988; 85: 6301-5.

14 Robinson B F, Collier J G. Vascular smooth muscle. $\mathrm{Br} \mathrm{Med}$ Bull 1979; 35: 305-12.

15 Dick W C, Jubb R, Buchanan W W, Williamson J, Whaley K, Porter B B. Studies on the sympathetic control of normal and diseased synovial blood vessels: the effect of nor $\beta$ receptor stim 133 xenon clearance technique. Clin Sci 1971; 40: 197-209. 